NOTE

\title{
Reasonable Polymer Component Number in Phase-Separation Computer Experiment on Polydisperse Polymer-Solvent System
}

\author{
Kenji KAMIDE and Yukio MIYAZAKI \\ Textile Research Laboratory, Asahi Chemical Industry Co., Ltd., \\ Hacchonawate, Takatsuki, Osaka 569, Japan.
}

(Received September 20, 1979)

\begin{abstract}
KEY WORDS Phase-Separation / Fractionation / Computer Simulation /
\end{abstract}
Since the late 1960s, Tung, ${ }^{1}$ Kamide and his coworkers, ${ }^{2}$ and Koningsveld et al. ${ }^{3}$ carried out in a very systematic and rigorous manner computer experiments on the phase-separation phenomena of polydisperse polymer-solvent systems and as a result, considerable accurate knowledge on this phenomena has rapidly accumulated.

To be two liquid phases (i.e., the polymer-lean phase and the polymer-rich phase) in thermodynamic equilibrium, the following conditions, originaly given by Gibbs, must be satisfied.

$$
\Delta \mu_{0(1)}=\Delta \mu_{0(2)}
$$

and

$$
\Delta \mu_{x(1)}=\Delta \mu_{x(2)}
$$

Here, $\Delta \mu_{0}$ and $\Delta \mu_{x}$ are the partial molar free energies of dilution of the solvent and that of the $X$-mer, respectively, and the subscripts 1 and 2 represent the polymer-lean phase and the polymer-rich phase, respectively. The explicit forms of $\Delta \mu_{0}$ and $\Delta \mu_{x}$ are given by the well-known relations in polymer dilute solution theory, such as those of Flory-Huggins or their revised theory, in which the concentration dependence of the polymer-solvent thermodynamic interaction parameter $\chi$ is taken into account:

$$
\chi=\chi_{0}\left(1+p v_{\mathrm{p}}\right)
$$

where, $\chi_{0}$ is the concentration-independent but temperature-dependent parameter, $p$ is the concentration dependence parameter and $v_{\mathrm{p}}$ is the polymer volume fraction.

Now, arrange all components differing in degree of polymerization $X$, and consisting of a polymer sample, in the order of increasing $X$ and define these components as the first, second, third, $i$ th $\cdots$ components from the smallest $X$ and write the $X$ of the $i$-th component as $X_{i}$. The number of components $N$ with different $X$, whose maximum is $X_{\max }$ and minimum is $X_{\min }$, constituting the sample, should be approximated with $X_{\max }-X_{\min }+1$. Consequently, theoretical calculations should be carried out for one eq 1 and $N$ eq 2's $\left(X=X_{\min } \sim X_{\max }\right)$. However, severe limitation on the calculation capacity in an electronic computer available at present does not always allow such a rigorous and ideal calculation. Actually, the $X$ value, which is considerably smaller than $X_{\max }$, is taken as the maximum $X$ (referred to as $X_{\text {max }}^{\prime}$ ) and the calculation is carried out for all components, but only a limited number of components $N^{\prime}\left(<X_{\max }^{\prime}\right)$ smaller than $N$ in the range $1 \leqslant X \leqslant X_{\max }^{\prime}$. Hereafter $N^{\prime}$ is termed the assumed component number. Therefore, the possibility for obtaining significantly different results may exist in two types of experiments: an ideal case $\left(N, X_{\min } \leqslant X \leqslant X_{\max }\right)$ and actual one $\left(N^{\prime}, X_{\min } \leqslant X \leqslant X_{\max }^{\prime}\right)$. Unfortunately, this point has not attracted much attention hitherto and has not been discussed in detail. ${ }^{4,5}$

This paper attempts to determine a minimum reasonable number of the components $N_{\mathrm{m}}$, sufficient for performing the computer experiments in phaseseparation with high accuracy. This is briefly defined as a reasonable polymer component number.

First, we assume that the molecular weight distribution (MWD) of the polymer samples is expressed by the Schulz-Zimm (SZ) or Wesslau (W) distribution, with the ratio of the weight-to the number-average degree of polymerization, 
$X_{w}{ }^{0} / X_{n}{ }^{0}=2$ and 5 and $X_{w}{ }^{0}=150-30000$ (the suffix 0 means the original polymer).

The computer simulation was carried out essentially according to the Kamide-Sugamiya procedure, ${ }^{6}$ using an electronic computer IBM System 370-158. In this case the double precision method was employed. In this paper, the following experiments were made under the conditions of the initial polymer concentration, $v_{\mathrm{p}}{ }^{0}=1.0 \mathrm{vol} \%$, the fraction size $\rho=0.001-0.99$, and $p$ (in eq 3 ) $=0$ : (1) single phase-separation experiment and (2) successive precipitational fractionation (SPF) and successive solutional fractionation (SSF).

In the above computer experiment, $X_{\max }^{\prime}$ was conveniently determined by,

$$
\begin{aligned}
& X_{\max }^{\prime}=10 X_{w}{ }^{0} \text { for the } \mathrm{SZ} \text { distribution } \\
& X_{\max }^{\prime}=20 X_{w}{ }^{0} \text { for the } \mathrm{W} \text { distribution }
\end{aligned}
$$

In eq 4, the weight of the components with $X>10$ $X_{w}{ }^{0}$ in the polymer sample is less than $0.01 \mathrm{wt} \%$ and in eq 5 , the corresponding value is less than $0.1 \mathrm{wt} \%$. Write the distance of the adjacent two $X \mathrm{~s}$, utilized in the calculation, as $D X\left(\equiv X_{i+1}^{\prime}-X_{i}^{\prime}=X_{i}^{\prime}-X_{i-1}^{\prime}, X_{i}^{\prime}\right.$ is the $X$ value of the $i$-th component, when all assumed components are numbered in increasing order of $X$. In general, $\left.X_{i} \neq X_{i}^{\prime}\right)$. Then, $N^{\prime}$ can be obtained directly as $X_{\max }^{\prime} / D X . X_{i}^{\prime}$ is also equivalent to $m D X\left(m=1,2,3, \cdots, N^{\prime}\right)$. The ratio of $X_{w} / X_{n}$ of the original polymer, calculated by using only assumed components, is referred to as $X_{w}{ }^{0} / X_{n}{ }^{0 \prime}$, in order to distinguish it from the true value.

The preliminary experiment shows that the minimum number of the components $N_{\mathrm{m}}$, satisfying the conditions given in eq 6 , is 300 for the $\mathrm{SZ}\left(X_{w}{ }^{0} / X_{n}{ }^{0}=2\right)$ sample and 500 for the $\mathrm{W}\left(X_{w}^{0} / X_{n}^{0}=5\right)$ sample.

$$
\begin{aligned}
& X_{w}{ }^{0} / X_{w}{ }^{0} \leqslant 1.00 \pm 0.03 \\
& X_{n}^{0 \prime} / X_{n}^{0} \leqslant 1.00 \pm 0.03 \\
& \left(X_{w}{ }^{0} / X_{n}{ }^{0 \prime}\right) /\left(X_{w}{ }^{0} / X_{n}^{0}\right) \leqslant 1.00 \pm 0.03
\end{aligned}
$$

These values are almost independent of $X_{w}{ }^{0}$. In the following experiments, we choose a SZ polymer sample with $X_{w}{ }^{0}=300$ and $X_{w}{ }^{0} / X_{n}{ }^{0}=2$ and a W polymer sample with $X_{w}{ }^{0}=300$ and $X_{w}{ }^{0} / X_{\mathrm{n}}{ }^{0}=5$ and $N^{\prime}>300(N=3000)$ for the former and $N^{\prime}>1000$ for the latter $(N=6000)$.

Figure 1 shows the effect of the fraction size $\rho_{\mathrm{p}}$ (the suffix $p$ means the precipitate) on the $N^{\prime}$ dependence of the partition coefficient $\sigma$, the volume ratio of the two phases $R$ and the ratio of $X_{w} / X_{n}$ of the polymers in both phases. In this figure, a $1.0 \%$ solution of the SZ polymer $\left(X_{w}{ }^{0}=300, X_{w}{ }^{0} / X_{n}^{0}=2\right.$ and $\left.N=3000\right)$ in a single solvent $(p=0)$ was fractionated. Evidently, $N^{\prime}>300$ gives true values for almost all $\sigma$, $R$ and $X_{w(2)} / X_{n(2)} ;$ these values are virtually equivalent to those obtained for $N^{\prime}=3000$. On the other hand, as $\rho_{\mathrm{p}}$ increases, the dependence of $N^{\prime}$ on

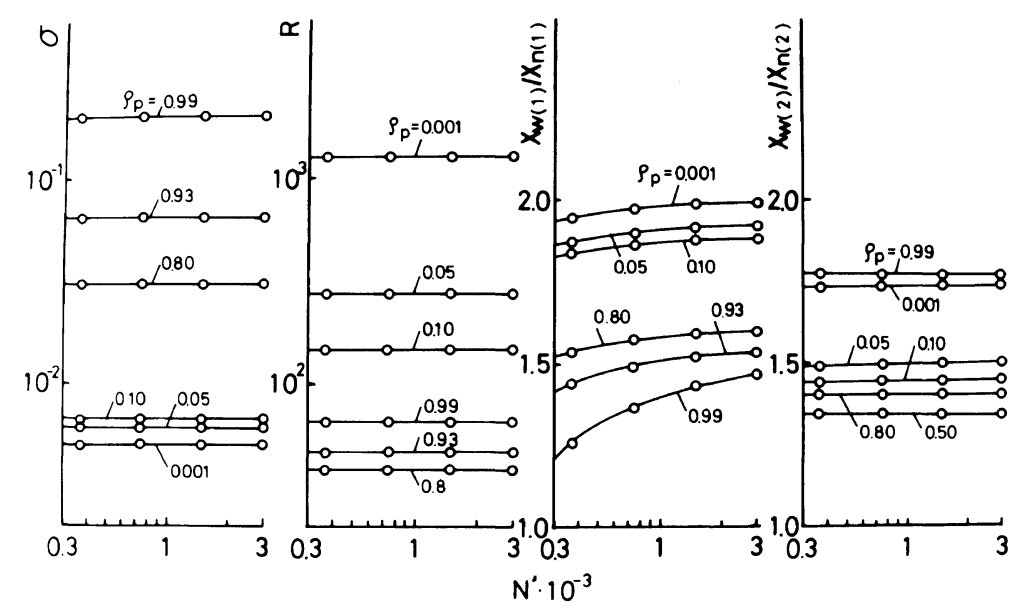

Figure 1. Effect of the fraction size $\rho_{\mathrm{p}}$ on the component number $N^{\prime}$ dependence of the partition coefficient $\sigma$, the volume ratio $R, X_{w(2)} / X_{n(2)}$, and $X_{w(1)} / X_{n(1)}:$ SZ polymer $\left(X_{w}{ }^{0} / X_{n}{ }^{0}=2, X_{w}{ }^{0}=300, N=3000\right) ; v_{\mathrm{p}}{ }^{0}=1.0$ $\mathrm{vol}^{\mathrm{o}} ; p=0$.

No. 11194 
$X_{w(1)} / X_{n(1)}$ becomes remarkable. That is, in the large $\rho_{\mathrm{p}}$ region, $N$ should be chosen as $N^{\prime}$. In conclusion, to calculate $X_{w} / X_{n}$ in the two phases within an error of $\pm 3 \%, N_{\mathrm{m}}=300$ for $X_{w(2)} / X_{n(2)}$ and $N_{\mathrm{m}}>1000$ for $X_{w(1)} / X_{n(1)}$ should be employed. The latter $N_{\mathrm{m}}$ value also depends on $\rho_{\mathrm{p}}$.

Similar phase separation experiments were carried out on the $W$ polymer $\left(X_{w}{ }^{0} / X_{n}{ }^{0}=5, X_{w}{ }^{0}=300\right.$, $N=6000)$. The results indicate $N_{\mathrm{m}}=1500$ for $\sigma, R$ and $X_{w(2)} / X_{n(2)}$, and $N_{\mathrm{m}}>3000$ for $X_{w(1)} / X_{n(1)}$.

Next, a $1.0 \%$ solution of the SZ polymer $\left(X_{w}{ }^{0} / X_{n}{ }^{0}=2, \quad X_{w}{ }^{0}=300, N=3000\right)$ in a solvent $(p=0)$ was fractionated by SPF or SSF into 20 fractions. In this case, $N^{\prime}$ was adopted as either 300 or 1500 . Figure 2 shows the relationships between $X_{w} / X_{n}$ of the fractions $\left(X_{w(2)} / X_{n(2)}\right.$ in SPF and $X_{w(1)} / X_{n(1)}$ in SSF) and their $X_{w}$. In SPF, two relations for $N^{\prime}=300$ and $N^{\prime}=1500$ coincide with each other, indicating that $N_{\mathrm{m}}=300$ is adequate in SPF. The final fraction (in this case 20-th fraction) is the polymer in a polymer-lean phase at the 19-th separation step and its $X_{w} / X_{n}$ being dependent of $N^{\prime}$, at least in the $N^{\prime}$ range of $300-1500 . N^{\prime}=300$ underestimates by $8 \%$ the $X_{w} / X_{n}$ value of the final fraction. Even in SSF, $N_{\mathrm{m}}$ is found to be 300, except for the first fraction. In this case, $X_{w} / X_{n}$ of the first fraction is $8 \%$ as small as the true value. In short, for the above polymer, $N_{\mathrm{m}}=300$ is obtained except for the lowest $X$ fraction.

In his calculation-fractionation study, Tung skillfully employed $D X=1$ in range $1 \leqslant X \leqslant 20$, $D X=3$ in the range $21 \leqslant X \leqslant 500, D X=30$ for $501 \leqslant X \leqslant 1700$ and $D X=300$ in range $1701 \leqslant X \leqslant 25700$, for an original polymer $\left(X_{w}{ }^{0}=840\right)$. This corresponds to $N^{\prime}=300$. Consequently, $D X$ becomes smaller in the lower $X$ region. Thus, as far as the component number is concerned, the reliability of his results may be quite satisfactory. Kamide et al. employed, in a series of their study, ${ }^{2} N^{\prime}=1500-6000$ (in their very early paper $N^{\prime}=800$ was used), which is, except for very special conditions, reasonable to assure the reliability of the results. The validity of the procedure of computer simulation used in this paper has been

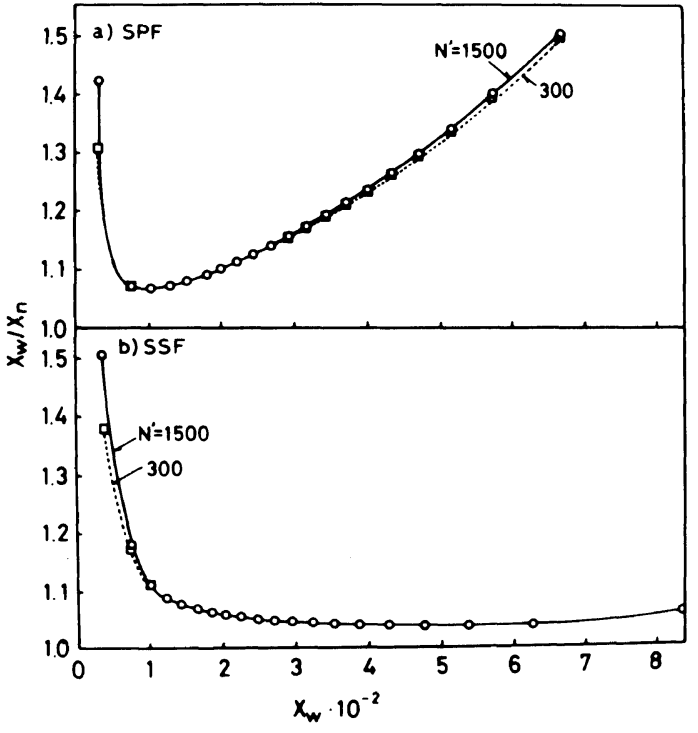

Figure 2. Relationships between $X_{w} / X_{n}$ of the fractions and their $X_{w}$, isolated by $\operatorname{SPF}(\mathrm{a})$ or $\operatorname{SSF}(\mathrm{b})$ : SZ polymer $\left(X_{w^{*}}{ }^{0} / X_{n}{ }^{0}=2, X_{w}{ }^{0}=300, N=3000\right), v_{\mathrm{p}}{ }^{0}=1.0 \mathrm{vol} \% ; p=0$; $n_{\mathrm{t}}=20$.

experimentally verified by us for systems such as polystyrene (PS)-methylcyclohexane, ${ }^{7.8} \quad$ PScyclohexane $(\mathrm{CH}),{ }^{7}$ and poly $(\alpha$-methylstyrene $)-\mathrm{CH} .{ }^{9}$

\section{REFERENCES}

1. L. H. Tung, J. Polym. Sci., 61, 449 (1962).

2. See, for example, K. Kamide, "Fractionation of Synthetic Polymer," L. H. Tung Ed., Mercel Dekker Inc., New York, N.Y., 1977, Chapter 2.

3. See, for example, R. Koningsveld, Adv. Polym. Sci.. 7, 1 (1970).

4. R. Koningsveld, private communication.

5. L. H. Tung, private communication.

6. K. Kamide and K. Sugamiya, Makromol. Chem., 139, 197 (1970).

7. K. Kamide, Y. Miyazaki, and T. Abe, Makromol. Chem., 177, 485 (1976).

8. K. Kamide, Y. Miyazaki, and T. Abe, Polym. J., 9, 395 (1977).

9. I. Noda, K. Kamide, Y. Miyazaki, and H. Ishikawa, Polym. Prepr., Jpn., 27, 558 (1978). 